\title{
Diseases that can be cured only by organ donations
}

\author{
Roxana Moscalu*, Anne Marie Smith, Harbans L. Sharma \\ Manchester Medical School, The University of Manchester, United Kingdom
}

\begin{abstract}
Nowadays, the failure of major organs, such as the kidneys, heart, lungs, liver, small bowel and pancreas caused by a significant number of diseases, put thousands of patients each year on the transplants list. But the breakthrough of blood vessels reconnection techniques and improvements in immunosuppressant, methods of organ conservation during transplantation, as well as in the post-operative care had a major contribution in considering organ transplant surgeries the best possible treatment for organ failure at the moment. The aim of this report is to present an overview on organ donation, focusing on the diseases for which the transplantation of an organ may represent the best possible treatment. As the NHS statistics reported, the number of patients who receive a chance for living a better life by organ donation is significantly increasing every year in the UK and the number of donors has been in a continuous rise too in the last years. Moreover, the statistics recorded by NHS showed that out of approximately 4600 transplants performed every year, more than 3000 are only kidney transplantations, making the kidneys the most donated organ. As mentioned before, organ transplantations are the best possible treatment for end-stage organ failure, which occur in severe cases of numerous types of diseases. Kidney transplantations are usually performed for patients with chronic kidney failure, whereas a heart transplant is considered the best possible treatment for heart failure, valvular heart disease, congenital heart disease, coronary artery disease and cardiomyopathy. Lung transplantations are most commonly used as treatment for end-stage related diseases such as cystic fibrosis, pulmonary edema, emphysema and pulmonary hypertension and pancreas transplantations are performed in cases of Type I insulin-dependent diabetes mellitus. Congenital liver defects and chronic liver infections are the main medical conditions that can be best treated by a liver transplant. Many years of laboratory research has now open the ways of successful small bowel transplantations which can save the lives of patients diagnosed with short bowel syndrome. However, as a result of the research that I have done that led to the finding of an impressive number of diseases that can be cured only by organ donation, my opinion is that organ donation should not be compulsory due to ethical problems or Human Rights (religious beliefs).
\end{abstract}

Keywords: transplant, organ, disease, organ failure, end-stage failure

\section{Introduction}

Organ transplantation is considered to be one of the most significant achievements of modern medicine, giving more than 3000 people every year, in UK alone, a chance for a better life. The donation of organs from one person to another is most commonly used in

Received: November 2015; Accepted after review: December 2015; Published: December 2015

${ }^{*}$ Corresponding author: Roxana Moscalu, Manchester Medical School, The University of Manchester, Oxford Road, Manchester, M13 9PT, United Kingdom.

Email: roxana.moscalu@student.manchester.ac.uk almost all cases of organ failure and is still known as the best treatment for it [1, 2].

In the following pages, general information about organ transplantation will be presented, as well as information regarding the diseases that can be cured only by organ donations.

\section{Organ transplantation}

\section{History}

One of the breakthroughs that opened the path of successful organ transplantation surgeries was the first demonstration of blood vessels surgical joining, performed by Doctor Alexis Carrel in 1902, which enabled the 
donated organ to be reconnected to a blood supply. Later, the development of blood-typing and anti-coagulants brought about the establishment of blood transfusions, which, as well, played a key role in making the organ transplantations possible [2].

All these led to the success of the first living kidney transplantation in 1954, when a patient's life was saved by his twin brother (the kidney was donated between twin brothers in order to reduce the risk of organ rejection by the receiver's body). In United Kingdom, the first living kidney transplant was performed in 1960, in Edinburgh. The donation occurred between identical twins and represented a total success as the donor was back at work three weeks later, and his brother returned to work after fifteen weeks [2].

Three years later the first liver transplant is performed, followed by the first kidney transplant from a decease donor in 1965 (which was performed in United Kingdom) and the first heart transplant in 1967 (it was performed in South Africa and the patient survived only 18 days, but the cause of death was pneumonia, which still led to the conclusion that it is possible for a patient to survive a heart transplant surgery). Only 1 year later, a heart transplant as well as a liver transplant are performed in the United Kingdom as well. Later on the first combined heart and lung transplant was performed, followed by a lung-only transplant three years later and a one lobe lung transplant in 1995 [2].

\section{Types of organ donations}

At the moment, there are three different types of organ donations: donation after brain stem death, after circulatory death or living organ donations.

Brain stem death occurs when a person has a permanent loss of brain stem activity, making it impossible for them to recover their consciousness or ability to breath without the support from a ventilator.

Donations after brain stem death are considered in cases when the potential donor has no brain stem activity which leads to permanent loss of consciousness and breathing capacity. When the damage is irreversible and the brain death is confirmed, organ donation can be preceded. If the patient had not registered as a potential donor, the relatives or partners are the ones who have to make the decision. In cases of donations after brain stem death, the most common organs transplanted are major ones such as heart and lungs [3].

Donations after circulatory death can be performed only when the potential donor's heart stops beating and the patient is declared death. In this case, the organs can quickly degrade and become unusable due to the decrease in the supply with oxygenated blood [4].

The last type of organ donations is the living organ transplantations. In these cases, the transplanted organ usually comes from a blood-related donor such as a parent, sibling or child, but can also be donated by a partner or an altruistic donor as long as the tissue type and the blood group match the ones of the receiver. The greatest percentage in living organ donations is occupied by kidney transplantations as the removal of one kidney will not jeopardise the donor's life if the remaining kidney is healthy [4].

\section{Organ transplantation statistics}

All the breakthroughs that have been occurring over the years led to an increasing number of organ transplantations and impressive figures regarding the number of donors and surgeries performed all over the world. According to the UK statistics for Organ Donation and Transplantation, between $1^{\text {st }}$ April 2013 and $31^{\text {st }}$ March 2014, 4655 organ donations were performed from 2466 living and deceased donors. Out of these, 3257 were only kidney transplantations, 924 were liver transplants, 261 pancreas transplants, 218 lung replacements, 206 heart transplantations and 26 bowel ones (Figure 1) [1]. Moreover, in the same time interval, more than 1 million people registered on the NHS Organ Donor Register, increasing the number of potential donors to more than 20 million. However, there is still a shortage of suitable 
donor organs as more than 6,800 patients are still on the waiting list for a transplant [1].

\section{Kidney transplantation}

Despite the little change that occurred in the actual techniques used in a renal transplantation, the continuous raise in receivers and donors' survival rates brought about by the improvements in dialysis, immune-suppressant drugs, antibiotics, the postoperative care and complications management have made the transplantation of kidneys the preferred treatment method for patients diagnosed with end-stage renal failure. However, this transplant procedure is not considered to cure the disease, but to offer a better chance of survival and a life lived as close to normal as possible [5]. In the UK, the patient survival rate at one year after kidney transplantation from a living donor is approximately $99 \%(95 \% \mathrm{Cl}$ : 98-99\%), and 95\% (95\% Cl: 95-96\%) after five years [6].

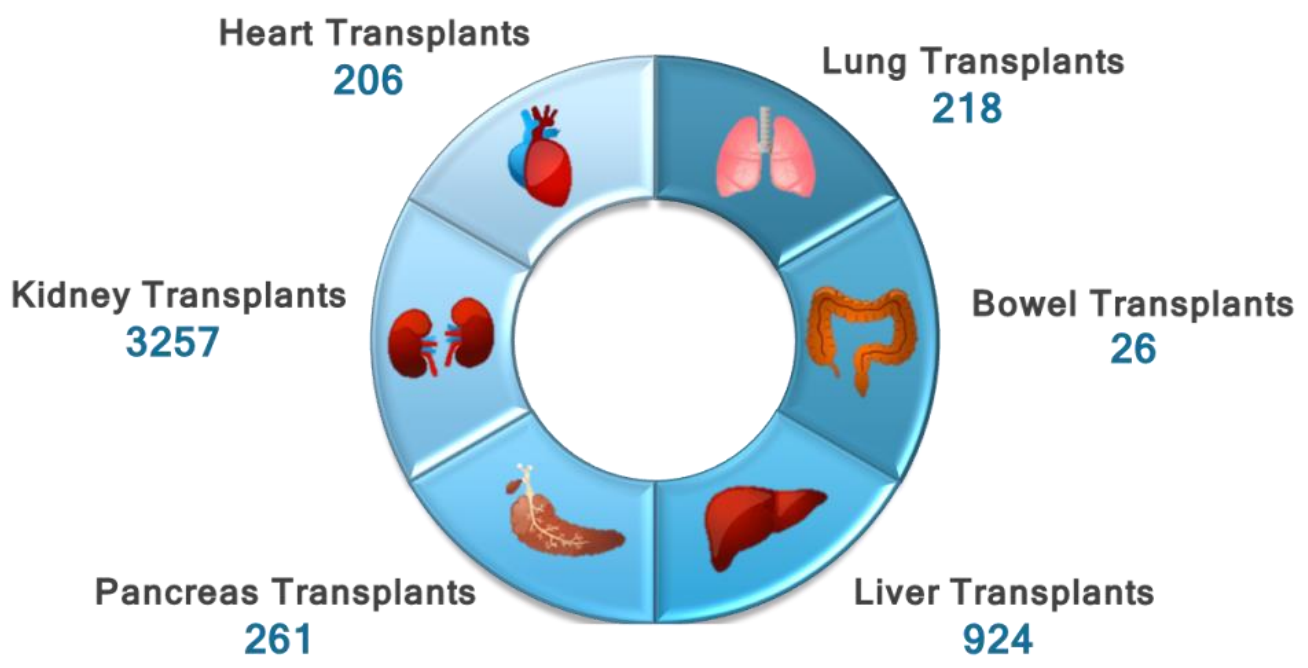

Fig. 1. Diagram showing number of organ transplantations between $1^{\text {st }}$ of April 2013 and $31^{\text {st }}$ of March 2014.

\section{Chronic kidney failure}

\subsection{Overview of the disease}

Chronic kidney failure is a rare (1 in 2000 people may develop it) long-term medical condition that involves the inability of the kidneys to complete their fundamental functions, including the removal of toxic wastes and excess water, involvement in maintaining the health and strength of the bones or in controlling blood pressure and the production of red blood cells.

The loss of kidneys' functionality is a gradual process, occurring over a long period of time (3 or more moths). In its final stages, it determines the development of end-stage renal failure (ESRF), a condition in which the kidneys stop operating almost completely and for which treatment involving dialysis (peritoneal dialysis or hemodialysis) and kidney transplant is indispensable for saving the patient's life. However, even after the transplant is successful, the patient is still considered to have the ESRF disease [7].

\subsection{Causes}

One of the most common causes of ESRF is thought to be Diabetes, which accounts for approximately $40 \%$ of the kidney failures in dialysis patients, especially in the cases where they have had the disease for 10 years or more, despite it being kept under control by use of insulin, tablets or a correct diet [8].

Another known cause of ESRF is a group of conditions called Nephritis, which are characterised by the long-term inflammation of the kidneys. Glomerulonephritis (damage of glomeruli) is one of the main sub-types that lead to the failure of the kidneys, despite following a treatment. One of its symptoms is a 
high blood pressure which is also associated with the chronic kidney failure as it damages the vessels and lowers the amount of blood that goes to the kidneys, causing their loss of function. Treatment for glomerulonephritis normally depends on the severity of the case, ranging from a change of diet (e.g. decreasing the amount of salt consumed in order to reduce the strain on the kidneys), taking medication for high blood pressure or the prescription of immunosuppressants if the disease is due to a problem with the immune system [9].

Polycystic kidney disease (PCKD) is an inherited disease with a $50 \%$ of being transmitted from parents to children. It involves the growth of multiple cysts in both kidneys, affecting their function and leading to ESRF. Normally, transplantation is required in order to replace a polycystic kidney.

Atheroma (atherosclerosis) is an accumulation or clot of cholesterol or other fats, which gradually blocks the arteries, diminishing the blood supply to various organs. When the renal arteries (arteries that transport blood to the kidneys) become narrowed, a deficit of blood to the kidneys is created, causing kidney failure. Most prone to the formation of atheroma patches are elders, but smoking can play a key role, significantly reducing the age at which this can occur.

However, some causes of ESRF still remain unknown in approximately $30 \%$ of the patients, who appear to have two small kidneys when an ultrasound is performed. The causes of the shrinkage are still unknown to the doctors who believe that something affected the development of the kidneys years before and the process of reducing in size has continued ever since [10].

\subsection{Symptoms}

Some of the symptoms for chronic kidney failure include itching skin, weakness, loss of appetite, poor sleeping, lack of concentration capacity, swollen ankles and feet due to fluid retention, muscle cramps and a continuous cold feeling in the body [11].

\subsection{Diagnosis}

The only method of diagnosis that is reliable for detecting this medical condition is a simple blood test known as the "estimated glomerular filtration rate" (eGFR) that measures the creatinine levels in the blood of the patient and, therefore, determines the health and functionality of the kidneys by assessing how much volume of blood can the glomeruli (filter the blood and form urine from waste products and excess water) filter in a specific period of time. Creatinine is a chemical waste product of muscle metabolism, transported in the bloodstream to the kidneys, where it should be filtered and then excreted in the urine. As the kidney function is lost, the levels of creatinine levels in the blood are increased and so kidney failure is detected. Normal levels of creatinine in the blood range between 45 and $120 \mu \mathrm{mol} / \mathrm{l}$; any slight elevation above $120 \mu \mathrm{mol} / \mathrm{l}$ is a clear indicator of kidney failure [10].

There has been created a scale indicating the stages of kidney function depending on the eGFR value (Tabel 1) [12].

Tabel 1. Stages of Chronic kidney disease [11]

\begin{tabular}{|c|c|c|}
\hline \multicolumn{2}{|r|}{ Stage of Chronic Kidney Disease } & $\begin{array}{c}\text { eGFR } \\
\mathrm{ml} / \mathrm{min} / 1.73 \mathrm{~m}^{2}\end{array}$ \\
\hline Stage 1 & $\begin{array}{l}\text { The e GFR shows normal kidney function but you are } \\
\text { already known to have some kidney damage or disease. For } \\
\text { example, you may have some protein or blood in your } \\
\text { urine, an abnormality of your kidney, kidney inflammation } \\
\text { etc. }\end{array}$ & $\mathbf{9 0}$ or more \\
\hline Stage 2 & $\begin{array}{l}\text { Mildly reduced kidney function and you are already } \\
\text { known o have some kidney damage or disease. People with } \\
\text { an eGFR of } 60-89 \text { without any known kidney damage or } \\
\text { disease are not considered to have chronic kidney disease. }\end{array}$ & 60 to 89 \\
\hline Stage 3 & $\begin{array}{l}\text { Moderately reduced kidney function. (with or without a } \\
\text { known kidney disease. For example, an elderly person with } \\
\text { ageing kidneys may have reduced kidney function without } \\
\text { a specific known kidney disease.) }\end{array}$ & $\begin{array}{l}45 \text { to } 59(3 A) \\
30 \text { to } 44(3 B)\end{array}$ \\
\hline Stage 4 & $\begin{array}{l}\text { Severely reduced kidney function. (With or without } \\
\text { known kidney disease.) }\end{array}$ & 15 to 29 \\
\hline Stage 5 & $\begin{array}{l}\text { Very severely reduced kidney function. This sometimes } \\
\text { called end-stage kidney failure or established renal failure. }\end{array}$ & Less than $\mathbf{1 5}$ \\
\hline
\end{tabular}




\subsection{Progress of disease}

According to the NHS statistics, between 2008 and 2009 in England, 4.1\% of the population aged 18 or above $(1,739,443$ people) were registered with chronic kidney disease (Figure 2) [13].

Onset kidney failure does not have a great impact on the general well-being of the patients as the kidneys start working harder in order to eliminate the excess of toxins and water, despite the fact that they are in a continuous process of degrading. Even if the rates of failure, as well as the symptoms may vary between different individuals, a great number of patients start to feel ailing when their kidney function is approximately $10 \%$ of normal and there is a rise in the creatinine levels up to $500 \mu \mathrm{mol} / \mathrm{l}$. Dialysis and transplants are necessary in the case of endstage kidney failure, a condition occurring at stage 5 of the chronic kidney disease, when the level of creatinine exceeds $800 \mu \mathrm{mol} / \mathrm{l}$ and the kidney's function drops down to only $5 \%$ [14].

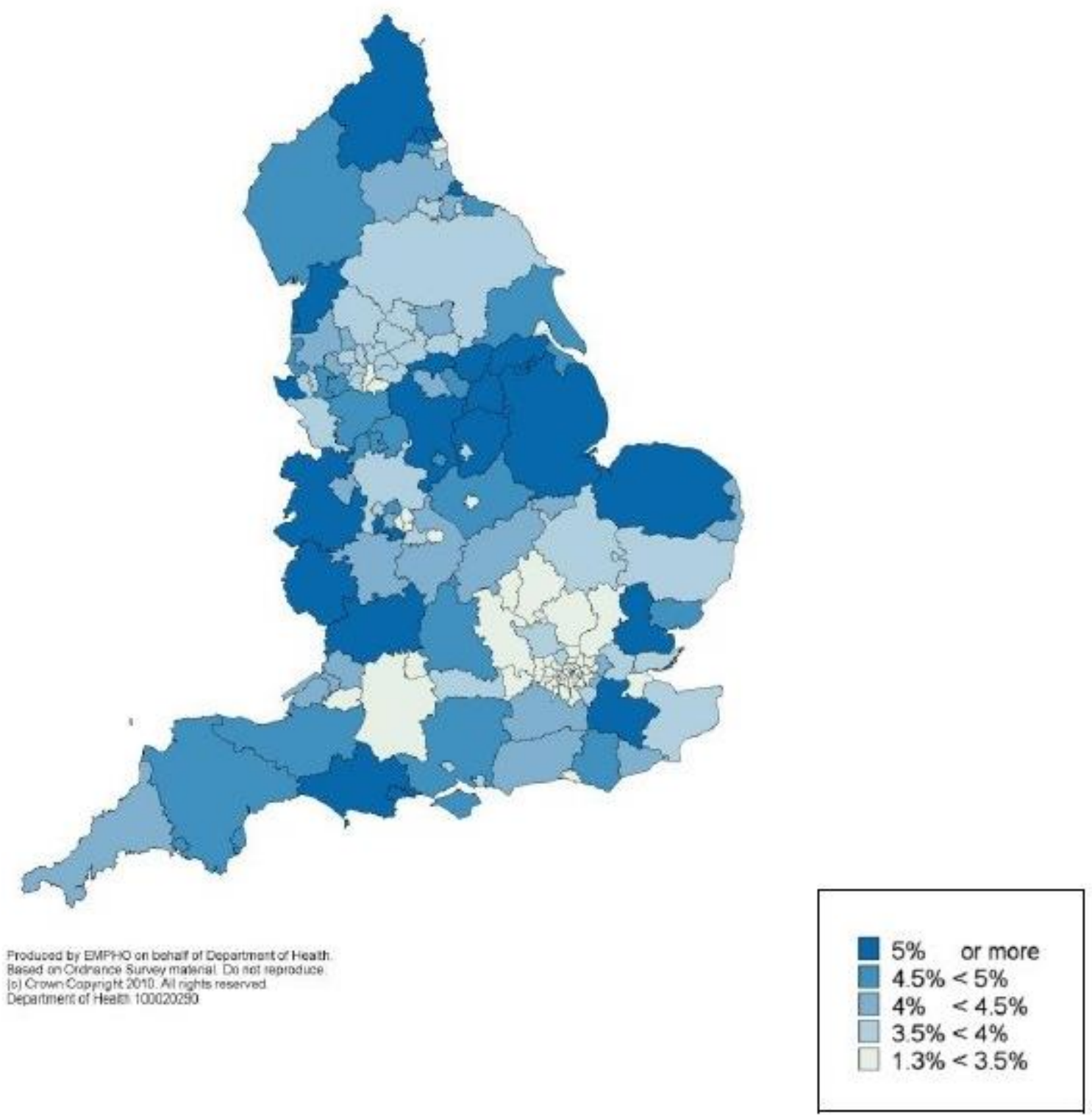

Fig. 2. Prevalence of Chronic kidney disease across England, between 2008 and 2009 [12] 


\subsection{Treatment}

The two treatments that offer alternative ways for the kidneys to perform their functions are dialysis, which can make up to $5 \%$ of the kidneys functions, eliminating most of the symptoms and allowing the patients to perform their everyday activities as close to normal as possible, and renal transplantation.

There are two different types of dialysis that can be used, known as peritoneal dialysis (happens inside the patient's body, involving the use of the patient's peritoneum as the dialysis membrane necessary for filtration), and haemodialysis (the traditional dialysis process which uses a machine, and, therefore, happens outside of the patient's body) [15].

\subsection{Renal transplantation}

The best treatment option for end-stage kidney failure is still considered to be the renal transplantation as it can provide the best quality of life by restoring approximately $50 \%$ of the kidneys functions and completely eliminating the need of dialysis. The only down sides of the renal transplantation are the possible incompatibility between the donor and the receiver and a life-long need of immunesuppressant drugs in order to avoid the rejection of the new organ. As long as the donor-receiver compatibility is checked, the only factors that can prevent the transplantation are serious cases of heart diseases or cancer. Age is not a solid influencing factor in the decision-making process as usually the transplantations are given to the patients on merit.

However, it is normally taken into consideration the fact that elders have a lower tolerance to the transplant surgery and the medication involved.

In order to be put on the national waiting list, patients have to first be stabilised by dialysis. Exceptions can occur, especially in the case of a patient with a failing transplant.

There are 3 types of transplants, depending on the sources from which the kidneys have been collected: cadaveric transplants (organs taken from a deceased person), living transplants (the organs can come from either a related or unrelated living person) and xenotransplants (research has been put into finding a possibility of transplanting organs from animals, but so far the risks involved are considered to be of great concern).

According to the NHS Blood and Transplant (NHSBT) UK statistics for Organ Donation and Transplantation, between 1st April 2013 and 31st March 2014 approximately one third of the total number of kidney transplants came from a deceased donor (Figure 3) [1].

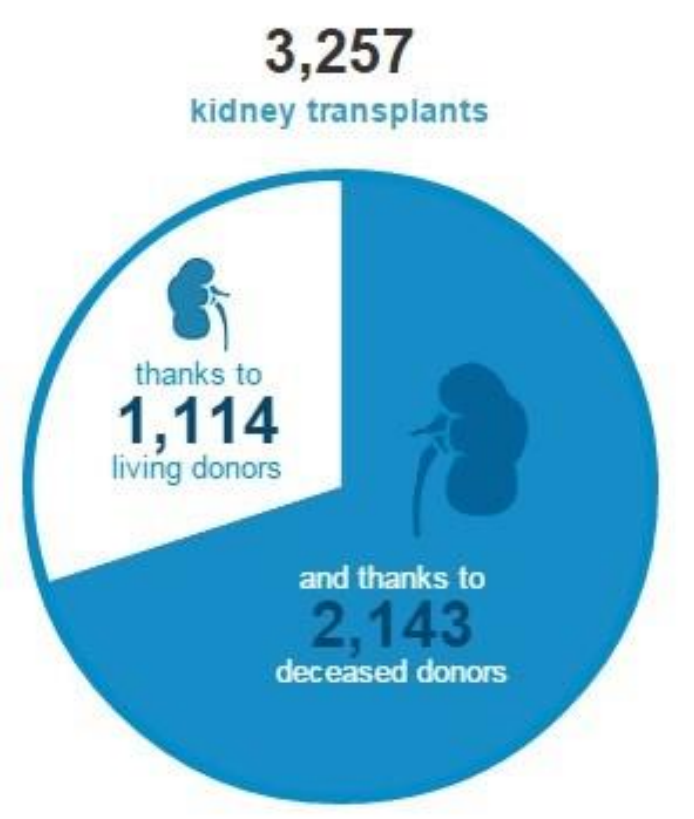

Fig. 3. Number of kidney transplants from a living or deceased donors between $1^{\text {st }}$ of April 2013 and $31^{\text {st }}$ of March 2014

Analysing the statistics over a long period of time, shows the figures in the case of deceased donor renal transplantations as recorded by the NHS Blood and Transplant at the end of each year, between 2004 and 2014 (Figure 4) [16].

While the number of donors and transplants performed has continuously increased over the 10-year period, the number of patients on the active transplant list raised significantly from 2004 to 2009 , and started to gradually decrease ever since. 


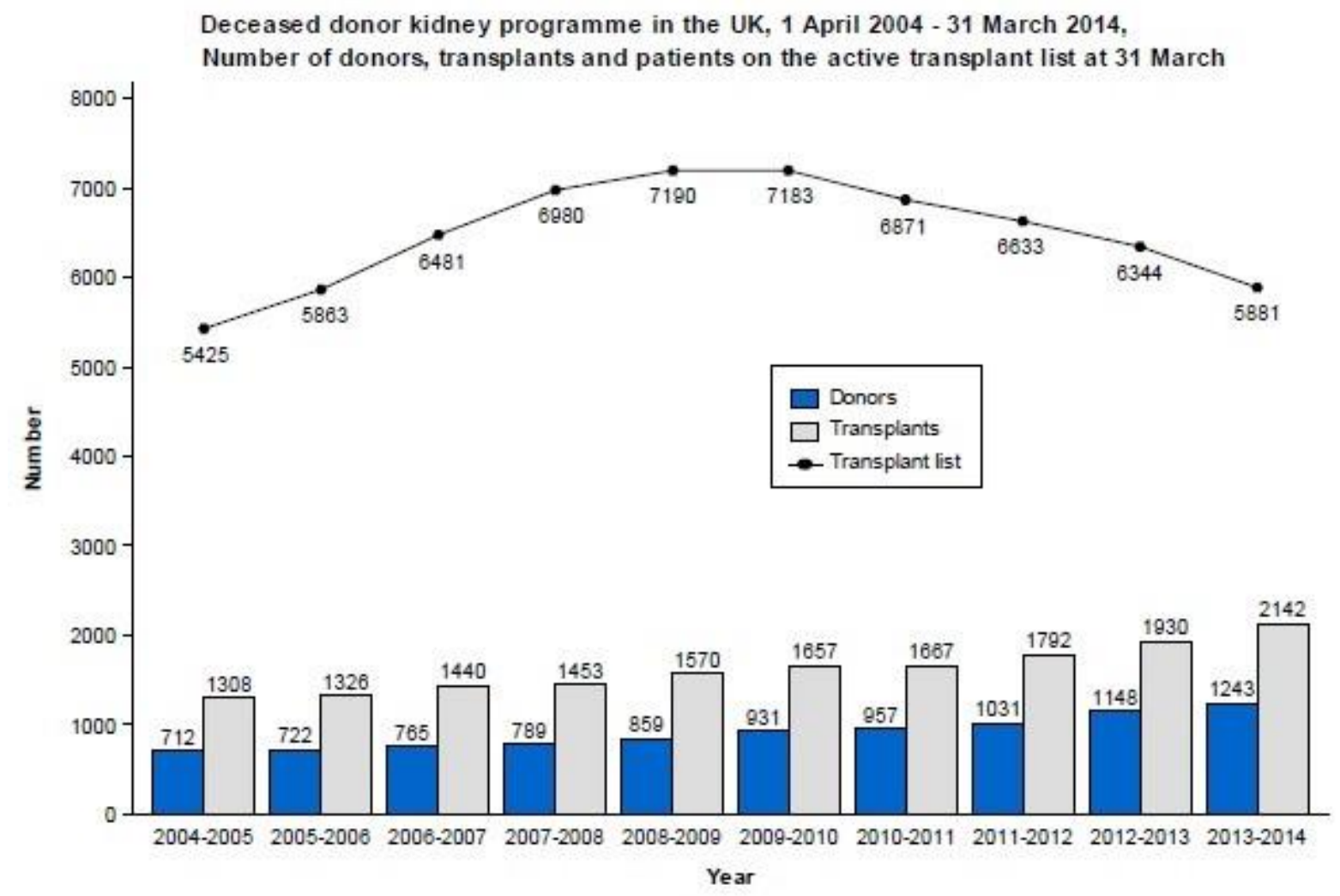

Fig. 4. Deceased donor kidney programme in the UK, 1 April 2004 - 31 March 2014, Number of donors, transplants and patients on the active transplant list at 31 March [15].

\section{Heart transplantation}

With approximately 35,000 heart transplants reported to the Registry of the International Society for Heart and Lung Transplantation and a growing number of 3,300 new procedures performed each year, heart transplantations have become the desired treatment method for patients with end-stage heart failure, registering a worldwide survival rate of more than $80 \%$ after 1 year and $60 \%$ after 5 years [17].

\subsection{The disease}

Heart transplantation is required when the heart is too damaged to perform its functions properly. This can usually happen in the case of 5 different conditions: congenital heart disease, coronary artery disease, heart failure, cardiomyopathy and valvular heart disease.

\subsection{Heart failure}

Heart occurs when the heart is not able to pump the blood normally and at the required pressure around the body.
The heart failure is usually caused by multiple conditions rather than a single heart problem; amongst these there can be identified the valvular heart disease, chronic artery disease, congenital heart disease, and cardiomyopathy.

Other causes include a high blood pressure or atrial fibrillation (irregularity of the heart rhythm), anemia or hyperthyroidism. Some of the main symptoms of a heart failure are shortness of breath, fatigue, and swallowing of the ankles. If the symptoms develop fast, the heart failure is considered to be acute, whereas if they appear gradually, the heart failure is described as chronic [18].

A balanced life, medication or devices (e.g. pacemaker) can help keep the condition under control.

However, in more severe cases or when the disease is caused by a problem of one of the components of the heart, transplantation is needed in order to replace the faulty organ and restore the normal functions of the heart [17]. 


\subsection{Valvular heart disease}

The valvular heart disease is represents a damage or defect in one of the four valves of the heart (the bicuspid or tricuspid valve, as well as the pulmonary and aortic ones), that prevents them from opening or closing completely [19].

The role of healthy valves is to assure the correct direction, power and timing of the blood flow in and out of the heart. However, when the valves are narrowed and hardened, they are called stenotic valves as they cannot entirely open, restricting or obstructing the blood flow and making the heart to pump harder in order to help the blood squeeze through the narrowing. On the other hand, when the valves cannot completely close, they are described as incompetent valves; this defect of the valves causes the blood to leak back in the chamber of the heart it comes from, stimulating the heart to overwork in order to pump the necessary amount of blood [18, 20].

Due to the strain put on the heart in both situations in order to pump the blood, the heat muscle becomes enlarged and thickened, which causes the loss of its elasticity and efficiency. Moreover, there is an increased risk of stroke or pulmonary embolism because of the high possibility of clotting for the blood that remains stagnant in one of the chambers [19].

The most common conditions that can cause heart valve disease are a congenital abnormality of the valves, rheumatic fever, cardiomyopathy (heart muscle's disease), heart damage due to a heart attack, prior endocarditis infection (bacterial infection of the endocardium), as well as advanced age. The disease can easily be prevented by following a healthy lifestyle: having a balanced diet, regular exercise, avoiding smoking, limiting the consumption of alcohol and correctly taking the prescribed treatment for other existent conditions such as diabetes or different heart diseases [18].

Even though the patient might not experience any symptoms, the most common ones include shortness of breath, swollen ankles and feet, fatigue, palpitations and chest pain, fever (when it is caused by endocarditis) and fast gain weight [19].
The diagnosis methods include, along with a check of the heart sounds (a murmur among the heart sounds can be an indicator of valvular heart disease), some tests such as an electrocardiogram (also called ECG or EKG, it measures the heart's electrical activity, the heartbeats' regularity, the thickness of the heart muscle and its possible damage), stress testing (patient has to exercise on a treadmill in a given period of time, in order to record his blood pressure, heart rate, changes in the EKG and breathing rates during physical activity), chest $X$-rays, cardiac catheterization (detects stenosis or incompetence of the heart valves by introducing a catheter into the heart chambers), or an echocardiogram (checks the size, shape and movement of the heart [19].

As the valves lose their functionality, the most appropriate treatment appears to be a valve surgery in order to repair or replace the damaged one, this way curing the disease. In mild cases, the disease can be kept under control by treatment with antibiotics in order to prevent possible infections or clot-preventing medication. In the case of a stenotic valve, a surgery called balloon dilatation can be performed to widen the valve by inflating a small balloon placed at the end of a catheter, through a blood vessel and at the narrowed spot [19].

\subsection{Congenital heart disease}

As mentioned in the name, congenital heart disease includes different types of defects of the heart that have been present since birth. In the UK, it has been reported that up to 9 in every 1000 babies can suffer from this disease [21].

The disease is usually caused by mutations of the genes or chromosomes, with higher risk of congenital heart disease occurring in situations where there is a family history of this condition, the mother has type 1 or type 2 diabetes or she had taken medication such as anticoagulants or antiepileptic during pregnancy, and when the baby is diagnosed with Down's syndrome [20, 22].

Some of the symptoms include fast heartbeat and breathing, inordinate sweating, tiredness and fatigue, or a blue undertone of the skin (cyanosis) [20]. 
Amongst the types of defects there can be found: septal defects (when 2 heart chambers are separated by a hole), coarctation of the aorta (narrowed artery), pulmonary valve stenosis and transposition of the major arteries (swap in positions of the pulmonary and aortic valves and the arteries they are connected to). These defects can be detected at various stages of life, from an ultrasound, to birth, childhood and even when the person is an adult [20].

If the defects don't threaten the patient's life, treatment is not needed; however, life counselling may be taken into account, during childhood and even adulthood. In the situation of a significant defect, surgeries including transplantations are required, as they can restore the functions of the heart almost completely [20].

\subsection{Coronary artery disease}

Coronary artery disease is a condition in which the arteries become blocked by a plaque of fat called atheroma, preventing the blood to flow normally through them. Annually in the UK, approximately 73,000 people die from this disease, the occurrence being higher in males (1 in 6), than in females (1 in 10). However, the NHS registered a number around 2.3 million people who still have the condition and are alive [23]. Along with an angina, the symptoms of the disease are the same as the ones for heart attacks and heart failure, but they have different manifestations in individuals and sometimes may not even occur until the disease is diagnosed with the use of tests (blood test and enquiries about lifestyle and family history), MRI or CT scan or a coronary angiography [22]. Even if the disease cannot be cured, the disease can be kept under control by treatment and other methods such as having a healthy lifestyle. In the case of coronary artery disease, a heart transplant is thought to be a good treatment option for restoring a normal life, considering that in the 1-year follow-up $85 \%$ of the transplants are still functioning normally, and around $70 \%$ after 5 years [22, 24].

\subsection{Cardiomyopathy}

Cardiomyopathy comprises of a number of diseases in which the muscle of the heart becomes stretched, thickened or stiff, making the heart unable to pump the necessary amount of blood needed [25].

There are four types of cardiomyopathy: dilated cardiomyopathy - heart muscle becomes stretched and thin, causing the inability of the heart to contract and pump the blood normally [24]; hypertrophic cardiomyopathy - thickening of the walls of the atria and ventricles and reduced chamber size, due to enlarged heart muscle cells; in UK 1 in 500 people are affected [24]; restrictive cardiomyopathy - stiffening and rigidity of the walls of the atria and ventricles, that prevents the chambers from relaxing [24]; arrhythmogenic right ventricular cardiomyopathy - death of the muscle cells (because of abnormalities of the proteins that hold them together) and the replacement of the affected tissue with a fatty and fibrous one [24].

With the exception of the hypertrophic cardiomyopathy, the other three types present the same symptoms as the heart failure (breathlessness, tiredness, swollen ankles and irregular heart rhythm. The arrhythmogenic right ventricular cardiomyopathy and the hypertrophic cardiomyopathy are the most common causes of unexpected deaths in young adults and children, the latter occurring especially for athletes [24].

There are numerous tests and scans that are performed in order to diagnose cardiomyopathy, including electrocardiogram, echocardiogram or even genetic tests if there is a family history of the disease [24].

Even if the disease cannot be cured, there are still treatments that can keep cardiomyopathy under control: lifestyle changes, medication (for high blood pressure or heart failure, beta-blockers to control the heart beats, anticoagulants or diuretics to prevent parts of the body to swallow from the excess fluid) or surgeries (injection of alcohol into the patient's heart in order to decrease the size of the muscle found in the septum of the heart, or a septal myectomy in which the same muscle's size is reduced by surgically removing a part of it. If the disease cannot be controlled by any of the previously mentioned treatments, a heart transplant is needed [24]. 


\section{Lung transplantation}

Along the years, laboratory research had a key role in introducing the lung transplantations among the successful procedures used to treat end-stage related diseases such as severe cases of cystic fibrosis, pulmonary edema, emphysema or pulmonary hypertension [26]. In cases of single-lung transplants the one-year survival rate is $78 \%$, dropping to $63 \%$ at three years, and $51 \%$ at five. If the median survival rate for patients with a single-lung transplant is 4.6 years, the outcome seems to be better for the double-lung transplant patients who have a 6.6 years median survival rate [27].

\subsection{Cystic fibrosis}

Cystic fibrosis is an inherited condition in which lungs and digestive system become clotted with thickened mucus and secretions produced by specific cells due to a mutation of the CFTR gene on chromosome 7 (this faulty gene causes the inability of these cells to properly handle sodium and chloride ions). In UK, approximately 1 in 2,500 babies are born with this disease and around 1 in 25 Caucasian people carry the gene, making it more common for them than for AfroCaribbean or Asian people. At the moment, in UK, there are more than 9,000 cases of cystic fibrosis recorded [28].

Symptoms may include a relentless cough, breathlessness, repeated infections in the chest and lungs etc. [29].

The disease can be diagnosed by 3 tests: a sweet test that evaluates the quantity of sodium and chloride ions in skin sweat; a genetic test that can identify the presence of the faulty gene; or a screening test performed 6 days after the baby is born in order to detect the presence in blood of a chemical specific for babies with cystic fibrosis, called immunoreactive trypsinogen [26].

When the cystic fibrosis affects the lungs, treatment can include physiotherapy and breathing exercise to help remove the thick mucus out of the airways, antibiotics and antifungals to find the possible bacteria found in the mucus, inhalers which open the airways and oxygen supplements. When the lungs are severely affected by the mucus, lung transplant is required. In rare situations, when the cystic fibrosis is attached surrounding tissues too, a combined heart and lung transplantation may be required. However, as cystic fibrosis is a genetic condition, it cannot be cured [26].

\subsection{Pulmonary edema}

Pulmonary edema is a common disease for elders ( 1 in 15 people between 75 and 84 years old and around 1 in 7 of the ones over 85 years), but more rare in youngsters, characterised by the presence of excess fluid in the alveoli of the lungs and preventing the absorption of oxygen in the bloodstream [30].

There are 2 main types of pulmonary edema. Cardiogenic (heart-related) pulmonary edema occurs when the pressure in the heart is raised above normal due to a weakened left ventricle that overworks in order to pump the blood that comes back form the lungs. Pressure starts to increase inside the heart from the left atrium and next through the lungs' capillary and veins; this makes the fluid to be transported into the alveoli through capillary walls. Diseases that might affect the strength of the left ventricle are coronary artery disease, hypertension, cardiomyopathy or valvular heart disease.

The second type of pulmonary edema, called noncardiogenic (not related to the heart) pulmonary edema, might be caused by an increased permeability of the pulmonary capillaries, or a leakage of fluid from these in the alveoli. The disease that influence the occurrence of this type are acute respiratory distress syndrome (characterised by a suddenly fill of the lungs with inflammatory white blood cells or fluid; can be caused by trauma, pneumonia, bleeding or systemic infection), disease of the nervous system (such as head injuries, seizures etc.), high altitudes (mainly the ones above 2,400 meters when the pulmonary capillaries might cause an increase of pressure), pulmonary embolism (develops when blood clots enter the pulmonary blood vessels), lung injury, viral infections, adverse drug reactions, toxins, inhalation of smoke or almost drowning [31].

Symptoms may include bloody coughing, breathlessness, difficulties in breathing while 
lying down, unconsciousness, anxiety, excessive sweating etc. [28].

Pulmonary edema's diagnosis process includes tests such as chest $\mathrm{X}$-rays, echocardiograms, electrocardiograms, blood tests or pulse oximetry (to measure the oxygen levels in the blood) [28].

Usually immediate treatment with medications and oxygen is required to remove the excess fluid in the alveoli, but the treatment can also depend upon the disease that caused pulmonary oedema. If the condition persists and complications occur, the pressure in the right ventricle can dramatically increase and possibly cause its failure [28].

\subsection{Emphysema}

Emphysema is a type of chronic obstructive pulmonary disease, characterised by a progressive damage to the lung's alveoli (air sacs put together in a shape resembling the structure of the grapes) which will mainly cause breathlessness. The condition occurs when the inner wall of the alveoli become weak and tear, leading to a decreased surface area inside the lungs as the multitude of air spaces become only one. Due to the damage of the alveoli, the air will not be able to be exhaled and let the space free for entrance of the new oxygen-filled air, which will cause, along with the reduced surface area, a deficit of oxygen in the blood flow [32].

The patients diagnosed with emphysema are more prone to acquiring heart problems (e.g.: "cor pulmonale", a condition characterised by an expanded and weakened section of the heart due to an increase pressure in the arteries between the heart and lungs), big holes in the lungs (empty spaces called "bullae" that can grow to up half of the lungs size), and collapse of the lungs [30].

The main cause of emphysema is smoking; other causes include exposure to pollution, factory fumes and marijuana smoke, as well as age. Apart from the shortness of breath, symptoms like blue colour of the lips and fingernails during physical activity or lack of mental awareness [30].

The disease can be diagnosed using different test such as chest X-rays, CT, blood tests (taken from an artery to measure the amounts of oxygen and carbon dioxide in blood), or measurements to determine the air capacity of the lungs using a spirometer (a device that the patient has to blow into) [30].

Treatment can include medication (bronchodilators which dilate the airways, inhaled steroids used to help eliminate the shortness of breath, or antibiotics), pulmonary and nutrition therapy, supplements of oxygen or surgery (damaged parts of the lungs can be removed surgically in order to allow the healthy tissue to expand and function efficiently). In the severe cases a single or double lung transplant can represent a nearcure of the disease [30].

\subsection{Pulmonary hypertension}

Pulmonary hypertension is caused by the increased blood pressure in the arteries that bring the blood to the lungs (pulmonary arteries). As the condition progresses, it can affect the arteries in the lungs, causing their thickening and, therefore, a decrease in their capacity to transport oxygen and carbon dioxide in and out of the blood. It can also have an impact on the right ventricle, as it will have to work harder in order to eliminate the deficit of blood to the lungs; this way, the muscle of the right ventricle weakens and, in the end, fails [33, 34].

Amongst the symptoms of the pulmonary hypertension there can be found breathlessness, tiredness, dizziness, angina (chest pain), raised heartbeat (tachycardia) and swallowing of the legs [32].

There are 2 different types of pulmonary hypertension. Primary pulmonary hypertension is an uncommon condition (that occurs more often in women than in men) with an unknown cause (it is thought that it is due to the spasms of the muscles of the pulmonary arteries), whereas the secondary pulmonary hypertension is caused by a different disease that alters the structure or normal functionality of the lungs, including chronic obstructive pulmonary disease, occupational lung disease or cystic fibrosis. Some other causes may include heart failure, HIV infection, obesity, diet drugs etc. [31].

Pulmonary hypertensions can be diagnosed using chest $X$-rays, an electrocardiogram, blood test (to measure oxygen levels) and a pulmonary function test 
to assess the damage done to the lungs. However, the best diagnosis method is measuring the pulmonary artery's blood pressure (using a wire catheter that will be inserted into the right side of the heart through a vein in the neck, arm or leg, in order to find out the blood pressure in the pulmonary artery as well as in the right ventricle.) [31].

Treatment can include medication for blood vessel dilatation (calcium channel blockers, nitric oxide or prostacyclin) or the elimination of excess fluid and oxygen supplies. However, in severe cases, a single or even double lung transplant is recommended in order to treat the problem that is causing the pulmonary hypertension [31].

\section{Liver transplantation}

With the first transplant being performed over 40 years ago, liver transplantation is nowadays a routine procedure that treats almost all types of end-stage liver failure. The number of transplantations performed worldwide had a dramatically increase, from only 330 surgeries in the 1980s (when the 1year survival rate was no more than $28 \%$ ), to over 52,000 after a decade (with a raised 1year survival rate of $80 \%$ ), out of which 24,564 were recorded only in Europe by end of 1996 [35]. Conditions that may require a liver transplant are congenital liver defects, chronic liver infections or damage caused by drug and alcohol abuse. Usually, the five-year survival rate for liver tranplant patients is aproximately $72 \%$. However, in cases of living donors, the outcome improves and the five-year survival rate raises to aproximately $78 \%$ [36].

\subsection{Congenital liver defects}

Congenital liver defects are rare conditions that normally affect the bile ducts, such as biliary atresia (obstruction of bile flow due to blockage or abnormal development of bile ducts) and choledochal cyst (obstruction of the bile flow due to malformation of the hepatic duct) [37].

The most common symptoms of the congenital liver defects are dark urine, jaundice (skin and eyes are yellow-coloured), pale, white or grey colour of the stools and abdominal mass and pain [34].

The process of diagnose can tests of the urine, blood, stool, liver enzymes and functions, liver biopsy, computed tomography (CT), ultrasound or MRI (magnetic resonance imaging) [34].

Treatment usually includes bile ducts reconstructive or bypass surgeries, and, in case of more severe cases, liver transplantation [34].

\subsection{Chronic liver infections}

Chronic liver disease includes 2 main conditions called Cirrhosis and Fibrosis (is a result of cirrhosis, that occurs when scar tissue starts to grow in the liver as a result of injuries, inflammations, infections or healing, affecting the normal functioning of surrounding organs) [38].

Cirrhosis occurs when the healthy liver tissue starts to be replaced by scar tissue, obstructing the blood flow thorough the liver and preventing it from functioning properly and making it unable to normally process substances like nutrients, drugs, poisons and hormones [35].

Along with alcohol, which is the most common, causes of cirrhosis can include drugs abuse, hepatitis, exposure to chemicals, glycogen storage disease, cystic fibrosis, bile duct obstruction, autoimmune diseases, heart and blood vessels diseases, diabetes, malnutrition etc. [35].

Cirrhosis symptoms depend mainly on the severity of the case and on the individuals. If a mild condition might not affect the patient in any way, a person experiencing more advanced cirrhosis might develop bloody vomit, gallstones, kidney failure, loss of muscle, lack of appetite and weight loss, hair loss, itching, curling of fingers, breast enlargement in men, weakness, hypertension, redness of the palms etc. [35].

Diagnosis methods for cirrhosis are physical examination, laboratory and liver function tests, CT, ultrasound, liver biopsy and cholangiography (contrast dye is introduced intravenously for a clearer bile ducts X-ray examination) [35].

Treatment depends solely on the individual and can prevent complication if it 
includes a healthy lifestyle with no drugs or alcohol abuse, taking vitamin supplements and proper management of any complications of the disease. In severe cases, liver transplantation is necessary [35].

\section{Pancreas transplantation}

Nowadays, Diabetes mellitus is the worldwide primary cause of adult kidney failure, amputations, blindness, impotence and amongst the main causes of childhood chronic diseases in poverty areas. Apart from a defective metabolism of glucose, the type I insulin-dependent diabetes mellitus syndrome (IDDM) is also characterised by microvascular complications resulted from hyperglycaemia. Even if a exogenously treatment with insulin prevents the occurrence of metabolic and microvascular complications when the right dose of insulin is provided, an endogenous secretion of insulin from a healthy pancreas is still considered the best way of carefully and continuously controlling the glucose levels in the blood throughout the day. Because of this, the best treatment for patients with IDDM is said to be the pancreas transplant as it can provide the best restoring of the pancreas functions, secreting the necessary amount of insulin like the healthy original gland, reducing the risks of secondary diabetic complications and offering the patient a chance for a normal life [39]. For the patients with a simultaneous pancreas-kidney transplant, $85 \%$ still have a functional pancreas after one year, but only $73 \%$ after five years. In pancreas-only transplant patients, the rates are $76 \%$ after one year and $53 \%$ after five [40].

\subsection{Type I insulin-dependent diabetes mellitus (Type 1 Diabetes)}

\subsubsection{Overview of the disease}

Type 1 Diabetes (IDDM) is condition that occurs quickly, especially in children and youngsters, when the glucose levels in the blood dramatically increase due to the stop of insulin (hormone that regulates the glucose levels in the blood) production in the body. According to the NHS records, in UK, type 1 diabetes accounts for $10 \%$ of the total number of diabetes cases [41] and it can be developed by 1 in 300 people [42].

\subsubsection{Causes}

IDDM is an autoimmune disease caused by a mistaken response of the immune system to the pancreatic cells: they are considered harmful by the immune system which will attack them [37].

\subsubsection{Symptoms}

When not enough insulin is produced in the body, the glucose is transported from the blood into the cells, where it will be used in order to produce energy. The lack of insulin will cause weight loss due to the breakdown of the fat and muscles of the patient's body, resulting in the development of diabetic ketoacidosis. Therefore, if the acidity of the bloodstream is increased, dehydration occurs [37]. This can explain the main symptoms of IDDM, which include continuous thirst, excess excreted urine and tiredness [38].

\subsection{Diagnosis}

The only reliable test that can diagnose IDDM is measuring the glucose levels in the blood, which will indicate a positive result if the values are high [38].

\subsection{Treatment}

Type 1 diabetes cannot be cured, but different treatment methods are used in order to maintain the levels of glucose in blood normal. These can include insulin injections, insulin pump therapy (insulin is pumped into the bloodstream using a device which inserts a small needle in the skin), islet cell transplantation (a transplant of healthy cells which produce insulin in the patient's pancreas) as well as a pancreas transplant [37].

\section{Small bowel transplantation}

A significant evolution of immunosuppressive medication have made possible in the last decade the successful transplantation of the small bowel, an organ considered unique and impossible to transplant due to its large size, its immunocompetent cells (that 
increase the risk of rejection of the donated organ), lack of sterility and tests that might provide a reliable follow-up internal examination [43].

Nowadays, the intestinal transplantation is only used for treating severe cases of small bowel syndrome.

\section{Short bowel syndrome}

\subsection{Overview of the disease}

When a large part (usually more than half) of the small intestine is removed, problems might occur in the absorption of nutrients, water and vitamins from food, causing a group of conditions called Short Bowel Syndrome. The bowel is comprised of the small intestine, where the food digestion and substances absorption takes place and which is divided into 3 sections called duodenum, jejunum and ileum, and the large intestine [44].

\subsection{Causes}

As previously mentioned, short bowel syndrome is caused by the removal of more than $50 \%$ of the small intestine as surgical treatment for injuries, intestinal disease or congenital defects. In adults, some conditions that might require eliminating a section of the small intestine are an inflammatory bowel disease known as Chron's disease, injuries caused by trauma or interrupted bloodstream, and cancer [40].

\subsection{Symptoms}

The leading symptoms encountered in the case of short bowel syndrome are diarrhea, which can have life-threatening effects such as dehydration, weight loss and malnutrition. Apart from these, the patients might experience cramps, heartburns, fatigue and bloating, as well as nutrient deficiencies contingent on the removed section of the small intestine: iron deficiency when the duodenum has been removed, malabsorbtion of the carbohydrates, proteins, fats and vitamins if the jejunum is missing and lack of bile acids and vitamin B12 when there is no ileum [40].

\subsection{Diagnosis}

There are numerous diagnosis methods that include laboratory tests (electrolytes test for dehydration, creatinine test to determine renal function, albumin test as indicator of nutrition status etc.), abdominal X-rays, ultrasounds and contrast computed tomography test, done densitometry or liver biopsy [45].

\subsection{Treatment}

The most important treatment to follow in case of short bowel syndrome is having the appropriate nutritional support which can include oral rehydration solutions, medications, parenteral nutrition (the necessary fluids, electrolytes and liquid nutrients are introduced into the bloodstream of the patient intravenously, using a tube inserted into a vein) and enteral nutrition (a feeding tube inserted into the stomach or small intestine ensures transport of liquid food). The treatment and recovery process is highly dependent on the severity of the case as well as the removed section of the small intestine, the length of the remaining organ and its capability to adapt to the new conditions. If complications of the parenteral nutrition occur (blood clots, liver failure, blood infections) or the initial treatment fails, intestinal transplant is required [40].

\section{Conclusion}

The research I have done led to the finding of numerous diseases that can be cured nowadays only by organ donations due to the successful breakthroughs of the last century. However, my opinion is that organ donations should not be compulsory.

It is every person's right to take any decision in regard with their body and, therefore, with their organs. Moreover, for a great number of people organ donations come in infringement with their religious beliefs and, as a result, they will decline any opportunity of becoming a potential donor. Furthermore, some ethical issues can arise regarding this subject. As part of my research showed a significant need of organs for patients on transplant waiting lists, I personally believe that, instead of making the organ donations compulsory, awareness should be raised in a more powerful way in order to inform the 
people about how important organ donations are nowadays and how many lives can these save each year, all over the world. But in the end, each person should be the one who individually decides to register as a potential donor.

\section{References}

1. Organ donations-Statistics [http://www.organdonation.nhs.uk/statistics available at 28/12/2015]

2. NHS-History of donation [http://www.nhs.uk/Tools/Pages/transplant.aspx available at Feb 24, 2015]

3. NHS Direct Wales-Encyclopedia. Brain death [http://www.nhsdirect.wales.nhs.uk/encyclopae $\mathrm{dia} / \mathrm{b} /$ article/braindeath available at 28/12/2015]

4. NHS. Organ donation [http://www.nhs.uk/conditions/Organ-

donation/pages/introduction.aspx available at 28/12/2015]

5. Hakim N, Danovitch G, Dausset J. Transplantation Surgery (Springer Specialist Surgery Series). Hakim N, Danovitch G, editors. United Kingdom: Springer-Verlag London, LLC; 2001, 135.

6. Blood and Transplant: Annual Report on kidney transplantation. United Kingdom, 2014.

7. Stein A. \& Wild J. Kidney Failure Explained. United Kingdom: Class Publishing; 1999, 1-4, 7.

8. Stein A. \& Wild J. Kidney Failure Explained. United Kingdom: Class Publishing; 1999, 6.

9. NHS-Glomerulonephritis [http://www.nhs.uk/conditions/Glomerulonephrit is/Pages/Introduction.aspx available at 28/12/2015]

10. Stein, A. \& Wild, J. Kidney Failure Explained. United Kingdom: Class Publishing; 1999, 5-6.

11. Stein, A. \& Wild, J. Kidney Failure Explained. United Kingdom: Class Publishing; 1999, 5.

12. Chronic Kidney Disease [http://www.patient.co.uk/health/chronic-kidneydisease-leaflet available at 28/12/2015]

13. NHS-Kidney Care. Kidney Disease: Key Facts and Figures. England, 2010.

14. Stein, A. \& Wild, J. Kidney Failure Explained. United Kingdom: Class Publishing; 1999, 6-7.

15. Stein, A. \& Wild, J. Kidney Failure Explained. United Kingdom: Class Publishing; 1999, 38, 48.

16. Kidney activity-NHS Blood and Transplantation [http://www.organdonation.nhs.uk/statistics/tran splant_activity_report available at 28/12/2015]

17. Hakim, N, Danovitch G, Dausset J. Transplantation Surgery (Springer Specialist Surgery Series). Hakim N, Danovitch G, editors. United Kingdom: Springer-Verlag London, LLC; 2001, 91.

18. NHS-Heart failure

[http://www.nhs.uk/Conditions/heart-

failure/Pages/Introduction.aspx available at 28/12/2015]

19. British Heart Foundation, Heart valve disease [http://www.bhf.org.uk/heart-

health/conditions/heart-valve-disease available at $28 / 12 / 2015$ ]

20. Johns Hopkins Medicine-Heart\& Vascular Institute. Vascular Heart Disease [http://www.hopkinsmedicine.org/heart_vascula r_institute/conditions_treatments/conditions/val vular_heart_disease.html available at 28/12/2015]

21. NHS-Congenital heart disease [http://www.nhs.uk/conditions/congenital-heartdisease/Pages/Introduction.aspx available at 28/12/2015]

22. British Heart Foundation. Congenital heart disease [http://www.bhf.org.uk/hearthealth/conditions/congenital-heart-disease available at 28/12/2015]

23. NHS-Coronary heart disease [http://www.nhs.uk/Conditions/Coronary-heartdisease/Pages/Introduction.aspx available at 28/12/2015]

24. Novartis. Diseases \& Conditions. Transplantation

[http://www.novartis.ca/en/disease_conditions/ $\mathrm{N}$-Z/Transplantation.html available at 28/12/2015]

25. NHS-Cardiomyopathy [http://www.nhs.uk/conditions/cardiomyopathy/ Pages/Introduction.aspx available at 28/12/2015]

26. Hakim N, Danovitch G, Dausset J. Transplantation Surgery (Springer Specialist Surgery Series). Hakim N, Danovitch G, editors. United Kingdom: Springer-Verlag London, LLC; 2001, 123.

27. National Heart, Lung, and Blood Institute-What are the Risks of Lung Transplant? [https://www.nhlbi.nih.gov/health/healthtopics/topics/lungtxp/risks availble at 28/12/2015]

28. Patient-Cystic Fibrosis [http://www.patient.co.uk/health/CysticFibrosis.htm available at 28/12/2015] 
29. NHS-Cystic

fibrosis

[http://www.nhs.uk/conditions/cystic-

fibrosis/Pages/Introduction.aspx available at 28/12/2015]

30. Patient-Cystic

Fibrosis

[http://www.patient.co.uk/health/pulmonary-

oedema available at Dec 1, 2015]

31. Mayo clinic-Pulmonary edema

[http://www.mayoclinic.org/diseases-

conditions/pulmonary-

edema/basics/causes/con-20022485 available at $28 / 12 / 2015$ ]

32. Mayo Clinic-Diseases and Conditions.

Emphysema

[http://www.mayoclinic.org/diseases-

conditions/emphysema/basics/definition/con-

20014218 available at 28/12/2015]

33. Cedars-Sinai. Conditions \& TreatmentsPulmonary Hypertension [http://www.cedarssinai.edu/Patients/Health-

Conditions/Pulmonary-Hypertension.aspx available at 28/12/2015]

34. NHS-Pulmonary hypertension

[http://www.nhs.uk/conditions/pulmonaryhypertension/Pages/introduction.aspx available at $28 / 12 / 2015]$

35. Hakim, N, Danovitch G, Dausset J. Transplantation Surgery (Springer Specialist Surgery Series). Hakim N, Danovitch G, editors. United Kingdom: Springer-Verlag London, LLC; 2001, 181.

36. Mayo Clinic-Liver transplant [http://www.mayoclinic.org/tests-

procedures/liver-transplant/basics/results/prc20014076 available at 28/12/2015]

37. Johns Hopkins Medicine-Congenital Liver Defects

[http://www.hopkinsmedicine.org/healthlibrary/c onditions/liver_biliary_and_pancreatic_disorder
s/congenital_liver_defects_85,P00665

available at 28/12/2015]

38. Johns Hopkins Medicine-Chronic Liver Disease/Cirrhosis

[http://www.hopkinsmedicine.org/healthlibrary/c onditions/liver_biliary_and_pancreatic_disorder s/chronic_liver_diseasecirrhosis_85,P00662 available at 28/12/2015]

39. Hakim N, Danovitch G, Dausset. Transplantation Surgery (Springer Specialist Surgery Series). Hakim N, Danovitch G, editors. United Kingdom: Springer-Verlag London, LLC; 2001, 211.

40. Mayo Clinic-Pancreas transplant [http://www.mayoclinic.org/testsprocedures/pancreastransplant/basics/results/prc-20014239 available at 28/12/2015]

41. NHS-Type 1 diabetes [http://www.nhs.uk/Conditions/Diabetestype1/Pages/Introduction.aspx available at 28/12/2015]

42. Patient-Type $1 \quad$ Diabetes [http://www.patient.co.uk/health/type-1-diabetes available at 28/12/2015]

43. Hakim, N, Danovitch G, Dausset J. Transplantation Surgery (Springer Specialist Surgery Series). Hakim N, Danovitch G, editors. United Kingdom: Springer-Verlag London, LLC; 2001, 235-236.

44. National Institute of Diabetes and Digestive and Kidney Diseases-Short Bowel Syndrome [http://www.niddk.nih.gov/healthinformation/health-topics/digestivediseases/short-bowel-

syndrome/Pages/facts.aspx available at 28/12/2015]

45. Patient-Short Bowel Syndrome [http://www.patient.co.uk/doctor/Short-BowelSyndrome-(SBS).htm available at 28/12/2015] 